\title{
Chlorotoxin-Indocyanine Green Imaging Agent BLZ-100
}

National Cancer Institute

\section{Source}

National Cancer Institute. Chlorotoxin-Indocyanine Green Imaging Agent BLZ-100. NCI

Thesaurus. Code C114979.

A tumor targeting imaging agent composed of the tumor-specific peptide chlorotoxin (CTX), a 36-amino acid neurotoxin found in the venom of the Leiurus quinquestriatus scorpion, linked to the fluorescent dye indocyanine green (ICG), with potential tumor imaging activity using a near-infrared (NIR) imaging system. Upon intravenous administration of BLZ-100, the CTX moiety of BLZ-100 specifically binds to and is internalized by cancer cells of neuroectodermal origin. Using a NIR imaging system, the ICG, which emits light in the NIR range, permits the intraoperative visualization of tumor cells. This leads to the clear distinction of healthy tissues from tumor cells, and facilitates the surgical removal of tumor tissue while sparing normal, healthy cells. 